\title{
The Character, Functions, and Styles of Instant Messaging in the Workplace
}

\author{
Ellen Isaacs, Alan Walendowski, Steve Whittaker, Diane J. Schiano \& Candace Kamm \\ AT\&T Labs, 180 Park Ave., Florham Park, NJ 07932 \\ ellen@izix.com,walendo@bitsmith.com, stevew@research.att.com, schiano@acm.org,cak@research.att.com
}

\begin{abstract}
Current perceptions of Instant Messaging (IM) use are based primarily on self-report studies. We logged thousands of (mostly) workplace IM conversations and evaluated their conversational characteristics and functions. Contrary to prior research, we found that the primary use of workplace IM was for complex work discussions. Only $28 \%$ of conversations were simple, single-purpose interactions and only $31 \%$ were about scheduling or coordination. Moreover, people rarely switched from IM to another medium when the conversation got complex. We found evidence of two distinct styles of use. Heavy IM users and frequent IM partners mainly used it to work together: to discuss a broad range of topics via many fastpaced interactions per day, each with many short turns and much threading and multitasking. Light users and infrequent pairs mainly used IM to coordinate: for scheduling, via fewer conversations per day that were shorter, slower-paced with less threading and multitasking.
\end{abstract}

Keywords: Instant messaging, workplace collaboration, informal communication, multitasking, media switching.

\section{INTRODUCTION}

Informal face-to-face (FTF) communication has been shown to serve many important functions in organizations, including complex coordination, problem solving, and social learning $[9,10,11,21]$. Early attempts to build tools to support informal communication focused on audio and video environments $[1,4,7,19]$. However, these attempts have not been widely adopted for several reasons, including the lack of support for core user tasks, cost, privacy concerns, and implementation difficulties $[1,9,10,20]$.

Instant Messaging (IM), in contrast, has become of great interest to the CSCW community because it is a tool that successfully supports informal communication $[5,6,13$, 14]. Nardi et al. [14], for example, reported cases where IM was preferred to informal FTF conversation because it is less intrusive and allows multitasking. Furthermore, IM has a huge base of users. Market reports indicate that over $140 \mathrm{M}$ people worldwide used IM at the end of 2000 [15]. In addition, IM is used by multiple populations for different

Permission to make digital or hard copies of all or part of this work for personal or classroom use is granted without fee provided that copies are not made or distributed for profit or commercial advantage and that copies bear this notice and the full citation on the first page. To copy otherwise, or republish, to post on servers or to redistribute to lists, requires prior specific permission and/or a fee.

CSCW '02, November 16-20, 2002, New Orleans, Louisiana, USA.

Copyright 2002 ACM 1-58113-560-2/02/0011 ..\$5.00 purposes: It has been widely adopted by teenagers for socializing, and by adults for both social and work purposes $[5,14]$. This suggests that IM merits detailed study. A better understanding of the properties of IM that enable it to support informal communication would help in the design of other novel technologies for supporting informal communication.

Most of our initial understanding of the use of instant messaging comes from self-reports, primarily interview studies $[3,5,6,13,14]$ or marketing surveys $[12,15,16]$. While helpful for getting a sense of IM practices, these studies usually have been based on relatively small sets of users and little direct observation of instant messaging activity. We were in the fortunate position of being able to collect a large sample of direct observations: over 21,000 IM conversations by 437 users. This should enable us to provide a more accurate picture of IM usage characteristics. We also evaluated these IM conversations qualitatively to refine our understanding of IM functions. We begin by summarizing previous findings.

\section{Current claims about IM usage}

Descriptions of IM from prior studies fall into three areas: (1) the character or properties of IM conversations, (2) the functions of $I M$, i.e. the tasks it is used to support, and (3) the pattern of IM use, i.e. how frequently people use IM and with whom.

Conversational Character. Three main observations are made about the character of IM, namely (a) that IM conversations are brief, (b) that media switching is prevalent, and (c) that multitasking is common while conversing in IM $[3,5,6,12,13,14,15,16]$. The first observation about the character of IM is that conversations are brief, addressing a single purpose. They focus on rapid exchanges (e.g. questions and answers) and brief interactions to coordinate conversations in other media (e.g. scheduling impromptu phone or FTF meetings). A few studies have mentioned one exception, noting that IM is sometimes used over long periods of time, with each person sending messages sporadically while primarily focusing on other activities [5, 14].

A second point about the character of IM is that media switching is prevalent $[3,14]$. Two main reasons for media switching are cited. First, the conversation becomes too complex to continue in IM, so participants agree to switch to phone or FTF interaction. For example, Connell et al. [3] stated, "Each new technology has its advantages and disadvantages. For example, instant messaging is quick and 
efficient for brief interactions, but when discussion becomes complicated, people often find themselves abandoning the chat and picking up the phone." Second, users switch from IM to another media when participants initiate an IM conversation to arrange an impromptu phone or FTF interaction [13]. In either case, the implication is that IM has limitations for anything more complex than simple and brief interactions.

Finally, multitasking is thought to be prevalent in IM. Several studies note that a major benefit of IM is that conversations can take place while carrying out other activities. Nardi et al. [14], for example, report that users liked that they could respond to quick IM questions from coworkers while engaged in another task, such as talking on the telephone, or processing documents or email. In other cases, IM was deliberately used to set up a parallel communication channel, e.g., to have a private side conversation during an audio conference. Descriptions of teenagers' use of IM also highlight multitasking, reporting how they carry on multiple simultaneous conversations while surfing the web, answering email, watching TV, or talking on the phone [5].

Functions. In their study of IM usage in the workplace, Nardi et al. [14] interviewed 20 workplace users and concluded that IM is used primarily for four functions, variations of which are frequently mentioned in other research $[3,5,6,12,13,15,16]$. These functions include (1) quick questions and clarifications, (2) coordination and scheduling work tasks, (3) coordinating impromptu social meetings, and (4) keeping in touch with friends and family.

The first category, quick questions and clarifications, is most commonly mentioned in other reports. IM is reported to be highly effective for asking quick questions of coworkers, who can respond rapidly without the overhead of telephone or FTF interaction. For example, IDC reports, "Users see IM as a medium for quick, semi-permanent 'flashes' that beg a near-immediate response" [12].

Other sources point to the role of IM in scheduling meetings or arranging impromptu discussions. IM is reported to be valuable in allowing remote coworkers to set up impromptu phone and FTF conversations [14]. Colleagues who share a physical environment can hold impromptu conversations when they encounter each other $[9,10,21]$. IM provides information about the availability of remote coworkers, enabling similar opportunistic conversations. Milewski \& Smith [13] found that a team of researchers frequently used IM to coordinate impromptu phone calls among members of the group.

Nardi et al.'s [14] third and fourth categories focus on the social uses of IM, which are echoed in other reports. IM is used to keep in touch with friends and colleagues and to arrange impromptu social meetings. The perception that IM is used primarily for this purpose has led employers to be circumspect about sanctioning the official use of IM at work. One market survey reported, "'Fear of losing employee productivity' was the greatest concern of businesses in regards to instant messaging" [15]. Another indicated that IM is used for "non-business chatting with colleagues, and this fact made even current users feel that IM was a 'less serious' business tool than email'" [12].

Patterns of Use. While there is some consensus about the primary functions and character of IM, less is known about patterns of IM usage. A few studies estimate how many people users have on their buddy lists (lists of contacts) and how frequently users exchange messages, but these vary. Reports on average numbers of buddies range from about 7 to 14 , with some users having as many as $30[5,12,16]$. In one survey, $81.3 \%$ of respondents reported using IM "daily," with the remainder using it "weekly" [15]. Another survey indicated that $90 \%$ used IM daily, and those users reported having an average of 3.2 exchanges per day [16].

More importantly, that report suggested that there are two types of users: "intense" users who exchange many messages per day and "mainstream" users who exchange a few messages per day. However, it did not address whether those two user groups use IM fundamentally differently. Research on email and voicemail suggests that frequency of use affects communication behavior $[17,18]$. Furthermore, previous work on FTF communication points to another potential factor: prior experience with a conversational partner $[2,21]$. We plan to investigate the effects of both these factors (frequency of IM use and frequency of pairwise interaction) on IM usage.

To summarize, early studies suggest the following:

- IM conversations have a specific character: they tend to be brief and cover a single topic, and both media switching and multitasking are prevalent. However, IM may also be used for longer, intermittent interactions between established coworkers and friends.

- IM is used for four main functions: quick question and clarifications, coordinating impromptu work-related or phone meetings, coordinating impromptu social meetings, and keeping in touch.

The goal of this paper is to examine these observations using a large data sample. A primary hypothesis is that both the functions and character of IM usage vary depending on pattern of use, which is affected by: (1) how often each partner used IM and (2) how often pairs interacted with each other.

\section{METHOD}

\section{System}

We examined IM conversations logged on a prototype instant messenger application called Hubbub [8]. Hubbub is similar to other IM products in that it has a "buddy list" that provides awareness of and access to potential conversational partners, and it supports near-synchronous text-based interactions between two users. Hubbub has other features that distinguish it from other IMs. Specifically, Hubbub users have a Sound ID (short tune) that their buddies hear each time they become active on their computer, enabling users to maintain a background 
awareness of their buddies. Hubbub also provides easily visible cues indicating each buddy's level and duration of activity. Hubbub also allows users to send "sound IMs," or brief canned musical sequences that have meaning (e.g. "hi," "cool!," "thanks"). And while in a conversation, each user can see whether their partner is typing in their IM window, has focus in the window, or has focus outside that window (corresponding to speaking, listening, and not paying attention). These features might affect some characteristics of IM conversations when compared to other IM systems, but they are unlikely to affect their functions.

\section{Users}

In August 2000, a small group AT\&T Labs professionals began using Hubbub as part of Hubbub's iterative development process. This initial group comprised 28 researchers, software engineers, administrative assistants, and managers who worked at one of four sites, two in New Jersey, one in California, with one person in Texas. A revised version of Hubbub was released to the public in late January 2001 (see www.HubbubMe.com for free download info). Hubbub was announced in an AT\&T company email, and demos were given at various venues, including customer events and a CHI conference. As a prerequisite for using Hubbub, all users agreed to have their messages logged. Interviews and the logs indicated that logging had little effect on discussion topics or style of interaction. People often said they forgot they were being logged, and we saw logs of sensitive topics, e.g. personnel problems, news that had to be released with careful timing.

During the 16 months period between August 2000 and November 2001, 1,031 people ran Hubbub at least once, but many just tried it out and never became regular users. Since our goal was to understand stable IM behavior, we included only users who ran Hubbub for at least five days (a business week) and had at least 5 conversations, leaving 437 users. We call this the full group of users. Their email addresses suggest that about half worked for AT\&T (all over the US); other users came from other .com, .net, and .edu domains, with small groups from Canada, Portugal, and Australia. Informal analyses of logged conversations suggest that most users were adults at work, but some used it from home and others from multiple locales (two workplaces or workplace and home). Some appeared to be teenagers, presumably at home. Many users seemed familiar with other IMs; they used standard IM jargon and etiquette, and sometimes mentioned other IMs.

\section{Measures}

We grouped these users' messages into units of conversations, which we defined as a sequence of messages in which no two messages are separated by more than 5 minutes. We then removed conversations between any pair of users who had fewer than five conversations overall, since some exploration of Hubbub's sound messages and other unique features often took place before interactions settled into a stable pattern. This left a corpus of 303,648 messages comprising 21,213 conversations between 692 pairs of users over the 16 months of the study. From these conversations, we computed a variety of quantitative measures to learn about their characteristics.

To understand whether IM behavior is affected by how frequently each partner used IM and how frequently the pair IMed with one other, we further divided the conversations. First, we classified Users into two groups, Heavy and Light. Heavy users were those who averaged 3 or more conversations per day (cpd), and Light users had fewer than 3 cpd with any partner. (We counted only those days in which they ran Hubbub and were active on their computer, which for many people excluded weekends.) We divided Pairs into two groups: Frequent and Infrequent. Frequent pairs averaged one or more conversation per day; Infrequent pairs had fewer than one cpd with each other. This gave us 6 groups: Frequent Pairs of Heavy-Heavy Users (H-H), Frequent Pairs of Heavy-Light Users (H-L), Frequent Pairs of Light-Light Users (L-L), and Infrequent Pairs of H-H, H-L, and L-L Users (see Table 1).

\begin{tabular}{|l|l|l|l|}
\hline Pair & $\begin{array}{l}\text { Heavy-Heavy } \\
\text { users }(\mathrm{H}-\mathrm{H})\end{array}$ & $\begin{array}{l}\text { Heavy-Light } \\
\text { users }(\mathrm{H}-\mathrm{L})\end{array}$ & $\begin{array}{l}\text { Light-Light } \\
\text { users }(\mathrm{L}-\mathrm{L})\end{array}$ \\
\hline Frequent & 8,846 & 2,570 & 2,015 \\
\hline Infrequent & 1,152 & 4,036 & 2,591 \\
\hline
\end{tabular}

Table 1. Number of conversations for each group compared in the IM analyses.

Given these definitions, $15.4 \%$ of our sample included Heavy users, averaging $5.1 \mathrm{cpd}$; the other $84.6 \%$ (Light users), averaged $1.0 \mathrm{cpd}$. For pairs, $21.0 \%$ were Frequent, averaging 2.4 conversations with one another per day; the remaining $79.0 \%$ (Infrequent pairs) averaged $0.3 \mathrm{cpd}$.

To learn what people were discussing in the conversations, we conducted a content analysis on a smaller sample of the data. For example, we evaluated whether the partners were using IM to resolve a quick question, whether the partners switched from IM to a different medium, whether the conversation included threading, and whether the partners discussed scheduling, a work issue, personal matters, and so on. For logistical reasons, the content analysis was done only on a subset of the initial group's conversations, which took place during the first 23 weeks of the study when the 28 AT\&T Labs users ran Hubbub as part of its iterative development process. The corpus for the initial sample included 61,833 messages exchanged between 138 pairs of users, comprising 3,096 conversations. Because content analysis is very time consuming, we coded 500 conversations from this initial sample, randomly choosing a representative portion of conversations from each pair of IM partners. We included 100 conversations from each category shown in Table 1, except there were no cases of Frequent pairs of two Light users in this sample. All conversations were assessed by two coders independently. The coders agreed to varying degrees on different measures, but overall, they agreed $94 \%$ of the time, and all categories had over $90 \%$ agreement.

In the next section, we discuss the character of the conversations, and how it varied for different patterns of 
use - noting when the data come from the full vs. initial group of users. Then we look at the functions of IM, again considering how that was affected by pattern of use. Finally, we discuss the implications of our findings.

\section{CONVERSATIONAL CHARACTER}

\section{Overall usage}

First, we examine the basic characteristics of instant messaging.

Frequency and Duration. Overall, the full group had an average of 6.2 people on their buddy list, and averaged 1.7 conversations per day. The range was broad, from $15.5 \mathrm{cpd}$ for the most active user to 0.1 for the least, and the distribution had a long tail of infrequent users. Participants were active on Hubbub an average of 43 days, with a range from 5 days (our minimum to be included in the sample) to 450 days, stretched out over the 16 months of the study.

Conversations lasted an average of 4 min 23 secs, with an exchange of 17.2 turns (or messages). Each turn consisted of an average of 13.5 words, with a turn gap of 24 seconds. These results are in keeping with data about other workplace interactions, which indicate that both phone and FTF conversations tend to be short, frequent, intermittent, and unscheduled $[9,21]$. Estimates of workplace FTF conversations range from 2 to 15 minutes $[10,21]$, and interactions in a lightweight desktop videoconference system averaged about 3 minutes [19]. So IM conversations are indeed short, as expected, but that may be the result of being impromptu rather than being in IM. Other impromptu forms of workplace interaction are comparable in duration.

Media switching. Many studies have drawn attention to the phenomenon of media switching, where IM is used to coordinate conversations in another medium. We present two examples of media switching to indicate how this works. In the first case, the purpose of the conversation is to set up the phone call. Bianca notices that Lars is idle and asks if she can call when he returns. (In all examples, all identifying information has been changed, but punctuation, capitalization, and typos are preserved.)

[13:38:19] Lars goes idle

[13:39:15] Bianca becomes active

[13:40:12] Bianca: Hi Lars, when you return and you have a minute, can I call you? I'd like to talk further about the website.

[13:41:34] Lars becomes active

[13:41:53]Lars: I'm here now... I actually was thinking of messaging you about it... so go ahead and give me a call.

[13:42:08] Bianca: OK. what's yr extension?

[13:42:12] Lars: 1234

In this second example, Keri calls someone to a prearranged meeting.

[13:30:41] Keri: time for the meeting!!!

[13:31:42] Keri: we are in trev's office.

According to the content analysis of the initial users' conversations, people did indicate they were switching from IM to another medium, but not especially often. In $15.6 \%$ of the conversations, users switched to phone $(6.8 \%)$ or FTF $(8.8 \%)$, and they switched to asynchronous media (email, Web, or fax) in another $2.2 \%$ of the conversations. Usually, they used IM to arrange an impromptu meeting ( $7.8 \%$ of conversations) or to call together the participants of a pre-arranged meeting (7.2\%). Rarely did they switch to another medium in mid-conversation when IM became inadequate $(2.8 \%)$, although they may have done so without mentioning it in IM.

So when people switched from IM, it was almost always when they intentionally used IM to initiate a meeting, not because IM became inadequate for their needs, as is commonly reported. This practice of using IM to call together the participants of a pre-arranged meeting is a function not mentioned in previous reports.

Multitasking. To see whether users were multitasking while messaging with others, we looked at the number of times they moved their focus out of the window during conversations. (Multitasking can happen without moving out of the window, but this is a good approximation.) Remember that Hubbub indicates when one's IM partner has moved out of the window, which may discourage people from doing so to avoid appearing rude, so our measure is likely to be lower than other IMs (which typically indicate only whether the other person is typing). The full group of users moved out of the window an average of 3.8 times per conversation, about once every 70 seconds, and in $85.7 \%$ of the conversations at least one person multitasked. (This does not include cases where one person sent a message and never got a response, but it does include cases when someone didn't get an immediate response, moved out of the window, and then returned when the response came, so it is a high estimate of attention switching.) These results indicate that even in the workplace, users were frequently doing other things while also carrying on IM conversations, despite knowing their partner could see them doing it.

That parallel activity could be related to the conversation, as when someone opens a document to find information requested by the other person. We found in the content analysis that $10.0 \%$ of the conversations within the initial group included discussions of content viewed in another application. It appears that the bulk of multitasking occurred because participants were doing unrelated activity while also chatting with a partner.

Conducting multiple simultaneous IM conversations was much rarer. Among the full group, only $4.3 \%$ of users' conversations overlapped at least one other. However, 77\% of users never overlapped conversations; among those who did, $9.7 \%$ of their conversations overlapped one or more others. The highest number of simultaneous conversations observed was 4 , with the vast majority being two at a time.

\section{Pattern of use}

Next we considered whether the character of the conversations varied depending on usage pattern, specifically based on how often each partner used IM and 
on how frequently the pair interacted. We found that there were two distinct styles of IM use.

The following two conversations illustrate the two styles. First is a conversation between two heavy IM users who interacted frequently. (Turns in curly braces indicate a sound instant messages, e.g. $\{$ bye $\}$ indicates the user played the sound IM for "bye.")

[20:08:44] Keri: oops, I just realized I hadn't sent out the message about getting things to me by saturday (it was buried under a slew of ppt and biz plan windows.

[20:08:54] Sara: yeah, I noticed that

[20:08:58] Sara: actually,

[20:09:05] Keri: so anyhow I just sent that, and also my first pass. [20:09:12] Sara: I modified the template a bit to allow more room for the Technology vs Program roadmap

[20:09:15] Sara: slides

[20:09:22] Sara: so I was going to resend it

[20:09:27] Keri: ok, I'll integrate that.

[20:09:43] Sara: Oh, and I was going to include my first pass, so let me send it to you, and then you can put it together

[20:09:50] Sara: and give feedback -- I don't think Roger's going to like what I have

[20:09:56] Sara: I'll do that now...

[20:09:59] Sara moves out of window

[20:09:59] Keri: but I also ended up putting in a slide after tech road map that is our year 1 and year 2 goals.

[20:10:08] Sara moves into window

[20:10:13] Keri: great. I'm going to go home and I'll get it there. my kitties are probably starving...

[20:10:13] Sara: oh, okay, that's good

[20:10:18] Sara: $\{$ okay\}

[20:10:21] Sara: $\{$ bye $\}$

[20:10:23] Keri: $\{$ bye $\}$

In less than two minutes, this pair exchanged 17 turns with short gaps between messages. In several places they thread their messages (as when Keri says "great" in response to Sara's "I'll do that now"). Some thoughts were broken into multiple turns, as when Sara sends three connected messages in a row.

Contrast this with the following conversation between two Light users who interacted infrequently. It includes 14 turns over more than 6 minutes, with longer turns and longer gaps between turns. Each message is a complete thought, and there is no threading.

[14:01:22] Bob: $\{h i\}$

[14:01:32] Bob: Have you learned anything adequate from this call?

[14:01:43] Sam: well, the note on teh [project] was interesting.

[14:01:56] Bob: what do you think the upshot is?

[14:02:09] Sam: sounds like they would be willing to consider funding a separate portion of the work [on the project].

[14:02:32] Bob: perhaps we should have a direct conversation with Nick about his views and how we can make it valuable for him?

[14:02:47] Sam: yes, that would be good.

[14:03:20] Bob: only q would be whether to include Jack or one of his folks (I think he referred to his org) and do this 3-way...

[14:03:33] Sam moves out of window
[14:03:43] Sam moves into window

[14:04:18] Sam: would need to fit with how Jack's org does new service development and trials. his team would probably be on the receiving end of anything commissioned by [our group]

[14:04:36] Bob: right

[14:04:48] Sam: and Rex should be brought up to speed on this

[14:04:58] Bob: right

[14:05:00] Sam moves out of window

[14:05:03] Sam moves into window

[14:05:13] Sam moves out of window

[14:06:43] Bob: I assume Dick and Patricia will set up some formal followup from this call, and we can participate in that and help them structure the followup on this item. We should suggest that rex be in the loop and we contact Nick and perhaps Jack's folks on this.

[14:06:47] Sam moves into window

[14:07:32] Sam: Yes, that sounds like the right flow of events. The [project] work could be done separately from the 'formal agreement'.

How often each partner used IM. Table 2 shows how conversation characteristics varied based on how often each of the partners used IM. (All results are based on analyses of variance of the full group data, except threading results are based on a Chi Square among the initial group.)

\begin{tabular}{|l|l|l|l|l|}
\hline Characteristics & $\begin{array}{l}\text { Heavy- } \\
\text { Heavy }\end{array}$ & $\begin{array}{l}\text { Heavy- } \\
\text { Light }\end{array}$ & $\begin{array}{l}\text { Light- } \\
\text { Light }\end{array}$ & All \\
\hline Duration* (mins) $^{*}$ & $4: 25$ & $4: 10$ & $4: 36$ & $\mathbf{4 : 2 3}$ \\
\hline \# Turns** $^{* *}$ & 20.1 & 15.7 & 13.1 & $\mathbf{1 7 . 2}$ \\
\hline \# Text turns & 18.3 & 13.7 & 10.8 & $\mathbf{1 5 . 3}$ \\
\hline \# Sound turns $^{* *}$ & 1.8 & 2.0 & 2.3 & $\mathbf{2 . 0}$ \\
\hline Words per turn $^{* *}$ & 12.5 & 14.1 & 15.0 & $\mathbf{1 3 . 5}$ \\
\hline Turn gap (secs) & 22 & 23 & 28 & $\mathbf{2 4}$ \\
\hline $\begin{array}{l}\text { Threading } \\
\text { (\% convs) }^{* *}\end{array}$ & 41 & 31 & 15 & $\mathbf{3 2}$ \\
\hline $\begin{array}{l}\text { Times moved out } \\
\text { of window }\end{array}$ & 4.1 & 3.7 & 3.3 & $\mathbf{3 . 8}$ \\
\hline
\end{tabular}

Table 2. Characteristics of conversations depending on how often each partner used IM. * $\mathbf{p}<.01, * *=p<.001$.

Pairs of Heavy IMers exchanged more turns than did pairs of Heavy-Light users, who exchanged more turns than did two Light users. Interestingly, conversations between two Light users lasted the longest compared to those between $\mathrm{H}-\mathrm{L}$ and $\mathrm{H}-\mathrm{H}$ users. This pattern occurs partially because pairs with two Heavy users typed fewer words per turn and more often threaded their conversations than did H-L pairs, who did so more than L-L pairs. As a result, interactions including Heavy users had shorter turn gaps than those between Light users.

On the other hand, Heavy users were more likely to multitask and less likely to send sound IMs (which are quick to send) than were Light users. These behaviors should have made for slower-paced interactions, but that was not the case. So conversations including Heavy users were more intense than those without Heavy users, in that they had 
more turns (especially text turns) in a shorter amount of time, despite users' multitasking during the conversations. (Again, Hubbub's indication of the other person's attention and typing activity may have reduced threading and multitasking. Some mainly Light users told us they disliked threading and used the information to determine when to type and when to wait for a response.)

How often the pair interacted. The characteristics of conversations were affected slightly differently by the frequency of the pairs' interaction. Table 3 shows how the same conversational properties differed for Frequent and Infrequent pairs. Frequent pairs interacted for longer periods at a stretch and used fewer words per turn than did Infrequent pairs. However, they had a longer turn gap even though they had more threading, probably because they moved out of the window more often. In fact, users were especially likely to multitask if the pair consisted of $\mathrm{H}-\mathrm{H}$ or H-L users, $\mathrm{p}<.001$, indicating that both factors affected multitasking. Surprisingly, the first message of a conversation was responded to more quickly when the pair was Infrequent than when it was Frequent $(28$ secs vs. 35 secs, $\mathrm{p}<.001)$. Perhaps politeness dictated responding more quickly when the message was from an infrequent partner.

\begin{tabular}{|l|l|l|l|}
\hline Characteristics & $\begin{array}{l}\text { Frequent } \\
\text { Pair }\end{array}$ & $\begin{array}{l}\text { Infrequent } \\
\text { Pair }\end{array}$ & All \\
\hline Duration $^{* *}$ (mins) & $4: 38$ & $3: 57$ & $\mathbf{4 : 2 3}$ \\
\hline \# Turns & 18.4 & 15.2 & $\mathbf{1 7 . 2}$ \\
\hline \# Text turns & 16.6 & 13.0 & $\mathbf{1 5 . 3}$ \\
\hline \# Sound turns & 1.8 & 2.2 & $\mathbf{2 . 0}$ \\
\hline Words per turn & 13.0 & 14.3 & $\mathbf{1 3 . 5}$ \\
\hline $\begin{array}{l}\text { Turn gap (secs) } \\
\text { \#* }\end{array}$ & 25 & 22 & $\mathbf{2 4}$ \\
\hline $\begin{array}{l}\text { Threading } \\
\text { (\% convs) }^{* *}\end{array}$ & 41 & 26 & $\mathbf{3 2}$ \\
\hline $\begin{array}{l}\text { Times moved out of } \\
\text { window }\end{array}$ & 4.1 & 3.3 & $\mathbf{3 . 8}$ \\
\hline
\end{tabular}

Table 3. Characteristics of IM conversations based on how frequently the pair interacted. ${ }^{* *} \mathbf{p}<.001$.

Other Characteristics. In the content analysis of the initial group's conversations, we also looked at other aspects of conversations, including openings, closings, and whether conversations were continued from previous Hubbub IMs that day. We found that IMs between Frequent pairs were much more likely to cover topics mentioned in a previous IM that day than were those between Infrequent pairs $(44 \%$ vs. $27 \%, \mathrm{p}<.001$ ), whereas there was no effect based on the frequency with which each partner used IM. (This effect occurred partially because Infrequent pairs less often had multiple conversations per day.) So just as with FTF interactions, people who frequently interacted over IM often discussed topics over the course of the day, updating each other as news developed [21].

Given that, we expected to find fewer explicit openings among Frequent pairs, since many conversations were continuations of others. Surprisingly, this was not so, perhaps because a greeting is also a way to get someone's attention, which was required with each new conversation. However, Frequent pairs exchanged fewer explicit closings than did Infrequent pairs (21\% vs. $36 \%$ of conversations, $\mathrm{p}<.01$ ), perhaps because they expected to IM more later. Also, Frequent pairs were more likely than Infrequent pairs to explicitly interrupt their conversations, usually because someone had entered the room or they had received a phone call $(17 \%$ vs. $8 \%$ of conversations, $\mathrm{p}<.01)$. This may be due to several factors. Since their conversations were longer, they had more opportunity for interruption, and since they interacted often, they may have been more willing to take interruptions.

Summary. What emerges is a picture of two styles of use. Heavy IM users had many, fast-paced interactions with many short turns in a short amount of time. They carried on several threads in the same conversation and frequently moved out of the window to do other tasks. Light IMers' interactions were longer and slower paced with longer turns, slower turn gaps, and less threading and multitasking. If two users interacted with each other often, they had longer discussions that more often covered topics they'd previously discussed in IM. They more often interrupted their conversation to handle other matters, and they were less likely to close their interactions. This story is similar to others told about close collaborators who work together in the same location. They tend to have many brief interactions that often follow up on previous issues, usually without openings or closings [9,21].

\section{FUNCTIONS OF IM}

We explored the functions for which people used IM in the content analysis of the initial group's conversations. We start by evaluating existing descriptions of IM, and then explore other functions.

\section{Simple questions and information}

As mentioned, a common impression of IM is that it is used for quick questions and clarifications. Rather than defining this category in terms of time, we tried to capture its essence by focusing on "simple questions and information," defined as conversations that covered a single topic and stayed focused on accomplishing a goal, ending when the issue was resolved. (Most such Simple conversations were also quick, i.e. fewer than 12 turns.) They could be initiated either with a question or information, but messages that got no response were excluded. The following is an example of a Simple question and information conversation. The partners accomplish their goal in just 43 secs with 8 turns.

[16:14:03] Lenny: Ricky-- did Peggy stop by with the disk yet?

[16:14:13] Ricky: yup.

[16:14:20] Lenny: great, thanks

[16:14:23] Lenny: $\{$ bye $\}$

[16:14:33] Ricky: i'll drop it off at your place on the way home

[16:14:37] Ricky: $\{$ bye $\}$

[16:14:38] Lenny: see youthen

[16:14:46] Ricky: \{okay\} 
A total of 139 conversations ( $27.8 \%$ of the sample) were coded as Simple questions and information. Nearly all of them were work-related: $91.4 \%$ work vs. $8.6 \%$ personal, which is $25.4 \%$ and $2.4 \%$ of the whole sample of conversations, respectively.

Conversations were more likely to be Simple and if they were carried out by an Infrequent pair than by a Frequent pair (Table 5) and if they included a Light user (Table 4). Frequent pairs with two Heavy users were the least likely to have Simple question and info conversations (14.0\%).

So IM was indeed used to handle Simple issues, but that was not the most common use. About $72 \%$ of the interactions were not Simple questions and information. In addition, Heavy IMers and Frequent IM pairs were even less likely to use IM this way.

\begin{tabular}{|l|l|l|l|l|}
\hline Function & $\begin{array}{l}\text { Heavy- } \\
\text { Heavy } \\
\text { Users }\end{array}$ & $\begin{array}{l}\text { Heavy- } \\
\text { Light } \\
\text { Users }\end{array}$ & $\begin{array}{l}\text { Light-Light } \\
\text { Users }\end{array}$ & Total \\
\hline Simple $^{* *}$ & 15.5 & 33.0 & 42.0 & $\mathbf{2 7 . 8}$ \\
\hline \hline Sched/Coord & 21.0 & 32.0 & 48.0 & $\mathbf{3 0 . 8}$ \\
\hline Work & 65.5 & 63.5 & 51.0 & $\mathbf{6 1 . 8}$ \\
\hline Personal ${ }^{* *}$ & 19.5 & 11.0 & 4.0 & $\mathbf{1 3 . 0}$ \\
\hline Saying "hi" & 9.0 & 3.5 & 2.0 & $\mathbf{5 . 4}$ \\
\hline No resp $^{* *}$ & 16.0 & 23.5 & 38.0 & $\mathbf{2 3 . 6}$ \\
\hline
\end{tabular}

Table 4. Percentage of conversations that included messages related to each function, based on how frequently each partner used IM $* *=\mathbf{p}<.001$. (Significance based on Chi-Square analyses.)

Whether a conversation focuses on a Simple question or piece of info is in principle independent of its function. We developed several broad categories of functions to capture what people were accomplishing in IM. Each conversation may have had more than one function. Tables 4 and 5 show the percentage of conversations that accomplished each of the functions, relative to how often the partners used IM (Table 4) and how often the pairs interacted (Table 5).

\begin{tabular}{|l|l|l|l|}
\hline Function & Frequent Pair & Infrequent Pair & Total \\
\hline Simple $^{*}$ & 19.0 & 33.7 & $\mathbf{2 7 . 8}$ \\
\hline \hline Sched/ Coord & 31.0 & 30.7 & $\mathbf{3 0 . 8}$ \\
\hline Work & 62.5 & 61.3 & $\mathbf{6 1 . 8}$ \\
\hline Personal+ & 17.5 & 10.0 & $\mathbf{1 3 . 0}$ \\
\hline Saying "hi" & 3.0 & 7.0 & $\mathbf{5 . 4}$ \\
\hline No response* $^{*}$ & 16.5 & 28.0 & $\mathbf{2 3 . 4}$ \\
\hline
\end{tabular}

Table 5. Percentage of conversations that included messages related to each function, based on frequency of pair interaction. $+\mathrm{p}<.05, *=\mathrm{p}<.01$.

\section{Scheduling and coordination}

We considered a conversation "scheduling or coordination" if it related to the coordination of the participants' activities, including meetings and appointments, right then or in the future. Note that scheduling and coordination conversations can also be Simple, since they can be singlepurpose and quickly resolved. The following is an example of a scheduling conversation. Carla establishes Margaret's availability and then schedules a time for a FTF meeting:

[11:03:34] Margaret becomes active

[11:04:00] Carla: so are you back from your meeting?

[11:04:07] Margaret: \{yes\}

[11:04:11] Margaret: do you have time now?

[11:04:19] Carla: can I come down in 10 minutes?

[11:04:22] Margaret: $\{y e s\}$

[11:04:24] Carla: $\{$ okay\}

[11:04:28] Carla: see you then

[11:05:00] Margaret: $\{$ okay\}

As shown in Table 4, 30.8\% of the conversations included a discussion about scheduling or coordination. Again, most were work-related, comprising $85.7 \%$ of all scheduling conversations ( $26.4 \%$ overall), with only $14.3 \%$ personal (4.4\% overall). Of all scheduling conversations, $39.0 \%$ were considered Simple. More of the work scheduling conversations were Simple $(40.1 \%)$ than were personal ones $(31.8 \%)$. In other words, when people discussed personal scheduling issues, they were more likely to discuss other topics as well, whereas work-scheduling conversations more often accomplished the goal and ended. Still, few scheduling conversations were personal.

Frequency of scheduling conversations varied depending on how often each partner used IM, but not on frequency of pair interaction. As Table 4 shows, two Light users were more likely to discuss scheduling and coordination than were H-L pairs, and far more than were H-H pairs. Both Frequent and Infrequent pairs had scheduling conversations about a third of the time (Table 5). However, Frequent pairs' scheduling conversations were more likely than Infrequent pairs' to be about personal issues $(9.5 \%$ vs. $1.0 \%, \mathrm{p}<.001$ ), and Light users were more likely to have work-related scheduling conversations than were Heavy users $(14.0 \%$ for $\mathrm{H}-\mathrm{H} ; 29.0 \%$ for $\mathrm{H}-\mathrm{L} ; 46.0 \%$ for $\mathrm{L}-\mathrm{L}$, $\mathrm{p}<.001)$. In other words, personal scheduling conversations were rare, but when they happened, they were more often between Heavy IM users and Frequently interacting pairs.

So IM was used more often for scheduling and coordination than for Simple questions and info, although often these discussions were Simple. Still, this category did not cover the largest percentage of the discussions.

\section{Social talk}

Despite the perception that IM is commonly used for social purposes in the workplace $[12,14,15]$, it was relatively rare for our users to chat about personal matters in IM. Overall, $13.0 \%$ of conversations included any personal topics, and only $6.4 \%$ were exclusively personal. In this example of a purely personal conversation, a married couple discusses plans for dinner, getting home, and doing errands.

[16:03:59] Trev: take out sushi sounds good to me [16:05:24] Leah becomes active 
[16:05:27] Leah: I think its a great idea too. are you going to ride home in this rain or are you coming with me. we still need to get [16:05:29] Leah: dana's money

[16:06:18] Trev: I am on the phone at the mo but I think that I will still ride unless it gets awful how much money do I need

[16:06:41] Leah: we just need 540 right?

[16:06:54] Trev: $\{y e s\}$

Heavy IMers were more likely to discuss personal matters than Light ones were, and there was a suggestion that Frequent pairs were more likely than Infrequent pairs to cover personal topics. However, for all groups, discussions of personal issues were relatively rare, even including conversations that covered both work and personal matters.

The main purpose of workplace IM: Talking about work If people were discussing scheduling only a quarter of the time and they rarely discussed social matters, what were they discussing most of the time? By far the most common purpose of these workplace IM conversations was to discuss or carry out work. Overall $62 \%$ of conversations were about work. We divided this category into three areas:

- Work Talk: Discussions that furthered the work activity of the two people involved.

- Doing Work: Carrying out a work activity in another medium while discussing it through IM

- Work-Related Talk: Discussions about any other work-related topic, including administrative issues, personnel, computer trouble, work that others were doing, company news, etc.

Here is a conversation considered Work Talk, since it furthered work on Jamie and Trev's project. In it, they decide what technology to use for their project:

[16:40:38] Jamie: Hi Trev!

[16:40:45] Trev: hi jamie

[16:41:09] Jamie: Just finished talking with Brett. Will write up some notes

[16:41:29] Trev: great what happened

[16:41:32] Jamie: Big question: we assuming IMAP for everyone or also POP3?

[16:42:57]Jamie: overall things are real ugly for POP3 and netscape, but may be doable under IMAP

[16:43:11] Trev: I dont see why we cant go w imap

[16:43:29] Jamie: as long as all your users are using it.

[16:43:42] Trev: right

[16:44:43] Trev: i'm on the phone can we talk later

[16:45:05] Jamie: this presents some challenges for me too--I don't have any IMAP accounts--more an enterpise type thing

[16:45:20] Jamie: sure. will send notes

[16:45:22] Jamie: $\{$ bye $\}$

[16:45:27] Trev: bye

The next example is Doing Work because Jamie and Alfie used IM to test a Web site.

[17:03:28] Jamie: Hi Alfie! Can I ask a favor?

[17:04:24] Jamie: I need someone to browse a certain webpage to see if it brings up a user/password window

$\cdots$

[17:10:17] Jamie: The URL is $<$ gives URL $>$
[17:10:32] Alfie: ok

[17:10:58] Alfie: trying it now

[17:11:02] Jamie: great! I can't find any one else to try this from either company I consult with!

[17:11:12] Alfie: :-)

[17:11:15] Alfie: it's thinking....

[17:11:26] Alfie: probably doing the dns lookup

[17:12:06] Alfie: hmm

[17:12:16] Alfie: if I go to /name/, it asks for a passwd

[17:12:29] Alfie: if I try /name/program.exe , it just sits there

[17:12:46] Alfie: Is this apache authentication?

[17:13:12] Jamie: no idea--all thru a web UI to my webhosting service.

[17:13:23] Alfie: hmm

[17:13:24] Jamie: mine is sitting there too now

[17:13:37] Alfie: let me try netscape instead of IE

[17:14:12] Alfie: weird - same thing

[17:14:28] Jamie: 1 sec-trying something...

The pair continued to debug the problem for another 26 messages over $4: 39$.

Any work topic that was not Work Talk or Doing Work was considered Work-related. Overall, $61.8 \%$ of all conversations involved any type of work. Most were either Work-related $(54.0 \%)$ or Work Talk $(49.8 \%)$. Many fewer were Doing Work through IM (12.0\%). Table 6 shows the percentages of each type of work discussion relative to all work conversations and to all IMs.

\begin{tabular}{|l|l|l|l|}
\hline & convs & $\%$ of work IMs & $\%$ of all IMs \\
\hline Work Talk & 154 & 49.8 & 30.8 \\
\hline $\begin{array}{l}\text { Work- } \\
\text { related }\end{array}$ & 167 & 54.0 & 33.4 \\
\hline Doing Work & 37 & 12.0 & 7.4 \\
\hline Any & 309 & 100.0 & 61.8 \\
\hline
\end{tabular}

Table 6. Frequency of types of work conversations. (Conversations could be in more than one category.)

Work talk was not affected by pattern of use. That is, most conversations across all groups related to work. Frequent pairs discussed work $62.5 \%$ of the time and Infrequent pairs did so $61.3 \%$ of the time (ns). Conversations between two Heavy users were about work $65.5 \%$ of the time, $63.5 \%$ for H-L, and $51.0 \%$ for L-L (ns).

Looking at the categories of work discussions, only Doing Work was discussed more by certain groups. Two Heavy users more often carried out work while discussing it through IM than any others $(13.5 \%$ of their conversations vs. $2.0 \%$ for $\mathrm{H}-\mathrm{L}$ pairs and $6.0 \%$ for $\mathrm{L}-\mathrm{L}, \mathrm{p}<.001)$. There was also a suggestion that Heavy users were more likely to have Work Talk than were Light users $(39.0 \%$ for $\mathrm{H}-\mathrm{H}$, $27.5 \%$ for H-L, $21.0 \%$ for L-L, p<.05).

Among this workplace community, then, the primary use of IM was to talk about or carry out work, which most often involved extended and complex interactions - only $26.5 \%$ of them were about Simple questions or info. This finding is important because it contradicts the common perception 
of IM as a tool primarily used for quick questions, scheduling, and social chitchat. The most common use complex work discussions - has been overlooked.

\section{Saying "hi"}

We found one other function of the IMs in our sample: just saying "hi," although it happened rarely (5.4\%). In some cases, the users exchanged the Hubbub sound IM for "hi," but more often they had a quick exchange, such as this one, which occurred when Lars heard Bianca's Sound ID:

[16:22:10] Bianca becomes active

[16:22:39] Lars: Hey!!!

[16:22:50] Bianca: Hi!

[16:23:03] Lars: Sorry... I just sent you email, then heard your tune, so I couldn't resist

[16:23:12] Bianca: OK, will check for it. Natalie and Beth say hi.

[16:23:23] Lars: Say hi to them for me!

[16:23:28] Bianca: will do!

[16:23:50] Lars: And let me know if you have problems [with the website] or if the instructions aren't clear.

[16:25:27] Bianca: OK, thanks Lars.

We did not include cases when someone said "hi" but received no response, since that was a common way to start an interaction. The number of "saying 'hi" conversations was small, but there was a suggestion that two Heavy IMers who didn't message each other much were the most likely to just say "hi" (13.0\% of their conversations). No other group did so more than $6.0 \%$ of the time $(p<.05)$. Hubbub may have prompted more "saying "hi" conversations than other IMs might because the Sound IDs made it easy to notice remote colleagues when they became available, and the "hi" sound IM was easy to send.

\section{No response and "sticky notes"}

Independent of their functions, we discovered that $23.6 \%$ of the initial group's "conversations" consisted of one person sending one or more messages and getting no response, at least within our five-minute conversation cutoff. Although this is good portion of the conversations, it is low compared with estimates of unanswered phone calls and attempts to start impromptu desktop videoconferences. In previous workplace studies, $62 \%$ of phone calls didn't connect with their intended recipient $[17,21]$, and $75 \%$ of attempts to start desktop videoconferences received no response [19]. The lower IM percentage may be because IM recipients could respond up to 5 minutes after receiving a message, whereas the other media require a response within seconds. Also, Hubbub offers awareness cues that indicate whether the other person is available. (Other IMs offer some awareness cues, but they are less extensive and precise.) The fact that Light users and Infrequent pairs were more likely to get no response to their first message (Tables 4 \& 5 ) indicates that people did learn to use this awareness info.

In a third of the cases when the recipient did not respond $(33.9 \%)$, the sender appeared to use IM as a sticky note. That is, they offered some information that the other person could act on without needing to respond. Some examples include, "ok, I'm done talking to Gita," and "Therz's an extra ham sandwich down here if u want it." This technique broadens IM's usefulness. Some users told us that they sometimes intentionally used IM as a sticky note rather than sending email because they knew it would be visible as soon as the person returned to their computer, and it would be easier to retrieve and respond to than voicemail, as reported in [14].

\section{DISCUSSION}

These findings suggest the need to re-examine certain beliefs about workplace IM use. Prior research on the functions of IM has emphasized its use for rapid singlepurpose exchanges, scheduling, and socializing. Our analysis suggests that these reports have over-emphasized those functions and greatly under-reported its use for extended work discussions, at least among workplace users. We also found that workplace IMs only occasionally turned to personal matters, and few were exclusively personal.

Previous research on IM character suggests that IM conversations are quick, and that multitasking and media switching are prevalent. We found that IM conversations were indeed short, lasting about $4 \frac{1}{2}$ minutes, but perhaps longer than expected, and comparable to other impromptu conversations, i.e. via FTF, phone, or desktop videoconferencing. We found that media switching was not especially common (16\% of conversations) and very rarely occurred when the conversation became too complex $(3 \%)$. Instead, when participants did switch media, they used IM deliberately to set up FTF or phone interactions or to call together participants of a meeting. People did frequently multitask while using IM ( $85 \%$ of conversations), as is often reported, but only about $23 \%$ of users carried on multiple simultaneous IMs and not that often (10\%).

We discovered two styles of use, which we call working together and coordinating. Those who work together use IM for a range of collaborative activities. They have multipurpose discussions, sometimes for scheduling, but more often covering a range of complex work (and sometimes personal) topics. Their conversations are more intense: with many short messages in a short period of time, often threading their messages. They are more likely to take interruptions and less inclined to close their conversations. Furthermore, the intensity of these interactions makes them different from the slow-paced, intermittent style of use mentioned in other studies [5, 14]. Heavy IMers and pairs who interact frequently tend to use IM to work together, and therefore, more IM conversations are of this style.

Those who coordinate have short, single-purpose conversations, often to schedule interactions in another medium. Their conversations are relatively slow-paced: with fewer, longer turns with a minimum of threading. They multitask less often than those who work together, but still do so frequently. They are less likely to interrupt their conversations and are more likely to formally end them. Light IM users and pairs who interact infrequently are most likely to use IM to coordinate, and so fewer conversations overall are of this style. 
How can we explain the discrepancies between our findings and those of prior studies? We believe the differences can be explained by three factors. First, some prior studies explored social IM use, primarily among teenagers, which is likely to be different from adults' workplace IM use. Second, although we found that most conversations were of the working together style, more people used the coordination style, so interviews of small numbers of workers may uncover more coordination-style attributes. Third, in self-report studies, people tend to focus on salient, novel behaviors (e.g., bypassing rounds of email to quickly resolve issues, having many simultaneous conversations), taking for granted the ordinary ones (e.g. carrying out work). Content analyses based on logs, on the other hand, can't capture users' intentions or non-computer-based events (e.g. multitasking without leaving the IM window).

We note two caveats. First, while our quantitative results are based on a large data set, our content claims come from a much smaller and more homogenous population. Second, certain results regarding the character of IM may have been affected by Hubbub's unique sound communication and enhanced awareness features, and so may vary from other IM products.

One design implication of these results is that, while it might help to make media switching easier by integrating IM with the phone, users might make use of such a feature less often than expected. People seem to be very effective at discussing complex work topics using just text. And since all users often multitask while IMing, it would be a mistake to tightly integrate IM with certain applications and make it difficult to move to others.

Our results also suggest that the characteristics of heavy IM use - multiple, brief, intermittent interactions per day with minimal closings - mirror the nature of impromptu pairwise interactions in other media $[9,10,21]$. This finding reinforces the notion that these are key characteristics of lightweight, unplanned interactions. Other tools intended to support it must be designed to support those characteristics.

Overall, these results help explain why IM is often used for informal workplace communication, and why it is sometimes preferred to FTF communication [14]. IM supports a broad range of uses: not only rapid, singlepurpose interactions, but also complex work activities.

\section{ACKNOWLEDGMENTS}

Many thanks to Dipti Ranganathan for all her work on Hubbub, Ron Brachman for his support of the project, Leysia Palen for valuable comments on the paper, and to all the participants in this study.

\section{REFERENCES}

1. Bly, S.A., Harrison, S.R. \& Irwin, S. (1993). Media spaces: Bringing people together in a video, audio, and computing environment, Communications of the ACM, 36:1, 28-47.

2. Brennan, S. E., \& Clark, H. H. (1996). Conceptual pacts and lexical choice in conversation. Journal of Experimental

Psychology: Learning, Memory, and Cognition, 22, 1482-1493.
3. Connell, J., Mendelsohn, G, Robins, R. \& Canny, J. (2001). Effects of communication medium on interpersonal perceptions: Don't hang up on the telephone yet!, Proceedings of GROUP '01, Boulder, CO, 117-124.

4. Fish, R., Kraut, R., Root, R. \& Rice, R. (1993). Video as a technology for informal communication, Communications of the ACM, 36:1, 48-61.

5. Grinter, R. \& Palen, L. (2002). Instant Messaging in Teen Life, Proceedings of $\mathrm{CSCW}$ '02, New Orleans, LA.

6. Grinter, R. \& Eldridge, M. (2001). y do tngrs luv 2 txt msg?, Proceedings of ECSCW '01, Bonn, Germany, 219-238.

7. Hindus, D., Ackerman, M., Mainwaring, S., \& Starr, B. (1996). Thunderwire: a field study of an audio-only media space, Proceedings of CSCW '96, Boston, MA, 238 - 247.

8. Isaacs, E., Walendowski, A. \& Ranganathan, D. (2002). Hubbub: A sound-enhanced mobile instant messenger that supports awareness and opportunistic interactions, Proceedings of $\mathrm{CHI}$ ' 02 , Minneapolis, MN, 179-186.

9. Isaacs, E., Whittaker, S., Frohlich, D. \& O’Conaill, B. (1997). Information communication re-examined: New functions for video in supporting opportunistic encounters, Video-Mediated Communication, Lawrence Erlbaum, 459-485.

10. Kraut, R.E., Fish, R.S., Root, R.W. \& Chalfonte, B.L.(1990). Informal communication in organizations: Form, function, and technology, in S. Oskamp \& S. Spacapan (Eds.), People's Reactions to Technology, Newbury Park: Sage, 145-199.

11. Kraut, R.E. \& Streeter, L.A. (1995). Coordination in software development, Communications of the ACM, 38:3, 69-81.

12. Mahowald, R. \& Levitt, M. (2000). Finding a Place: Corporate Instant Messaging Market Forecast \& Analysis, 2000-2004, IDC Report.

13. Milewski, A. \& Smith, T. (2000). Providing presence cues to telephone users, Proceedings of CSCW '00, Philadelphia, PA, 89-96.

14. Nardi, B., Whittaker, S. \& Bradner, E. (2000). Interaction and outeraction: instant messaging in action, Proceedings of $\mathrm{CSCW}$ ' 00 . Philadelphia, PA, 79-88.

15. Radicati Group (2001). Instant Messaging and SMS, Market Trends 2001-2004. Radicati Market Report.

16. Rhinelander, T. (2000). Intense users will drive increased IM capabilities, Forrester Technographics Brief.

17. Rice, R. E. (1988). Issues and concepts in research on computer-mediated communication systems. Communication Yearbook. J. A. Anderson, Sage. 12: 436-476.

18. Sproull, L. \& Kiesler, S. (1991). Connections MIT Press: Cambridge, Mass.

19. Tang, J., Isaacs, E., \& Rua, M. (1994). Supporting distributed groups with a Montage of lightweight interactions, Proceedings of CSCW' 94, Chapel Hill, NC, 23-34.

20. Whittaker, S. (1995). Rethinking video as a technology for interpersonal communications: theory and design implications. International Journal of Man-Machine Studies, 42[5], 501-529.

21. Whittaker, S., Frohlich, D. \& Daly-Jones, W. (1994). Informal Workplace Communication: What is it Like and How Might We Support It?, Proceedings of CHI '94, Boston, MA, 131-137. 